\title{
Availability of highly sensitive troponin assays and acute coronary syndrome care: insights from the SNAPSHOT registry
}

\author{
Use of hs \\ troponin testing \\ of patients \\ hospitalised \\ with possible \\ ACS was \\ associated with \\ an increased \\ rate of non- \\ invasive cardiac \\ investigations \\ and fewer \\ inhospital \\ adverse events
}

Louise Cullen MBBS, FACEM!

John K French

MBCHB, PHD, MSC ${ }^{2.3}$

Tom G Briffa

Julie Redfern BSc, BAppSc, PhD ${ }^{4}$

Christopher JK Hammett BHB, MBChB, MD

David B Briege MBBS, PhD, FRACP5

William A Parsonage

FRACP'

Jeffrey Lefkovits MBBS, FRACP, FCSANZ ${ }^{6}$

Chris Ellis

$\mathrm{BM}^{7}$

Carolyn Astley $\mathrm{RN}, \mathrm{BN}, \mathrm{DrPH}{ }^{8,9}$

Tegwen Eleanor Howell BEcon, MEcSt ${ }^{4}$

John M Elliott MBChB, PhD, FRACP10

Derek P B Chew FRACP, FCSANZ, FACC"
$\mathrm{R}$ ecent community campaigns on the warning signs of a heart attack have significantly increased the number of patients presenting to emergency departments (EDs) with possible acute coronary syndrome (ACS). ${ }^{1}$ Concomitantly, the advent of assays with improved sensitivity for detecting circulating cardiac troponins (cTn) - which are fundamental to the diagnosis of acute myocardial infarction (AMI) - has allowed the detection of low concentrations of these biomarkers, ${ }^{2}$ and an increase in proportions of ED patients with AMI has been reported. ${ }^{3}$

There has also been an associated increase in the number of patients with an elevated cTn level that is not attributable to an unstable coronary artery plaque resulting in an $\mathrm{ACS}^{3} \mathrm{It}$ is recommended that patients in the ED who have an elevated troponin level have further assessment and/ or management of the underlying cause, which often includes admission to hospital with resulting use of resources. ${ }^{4}$ Thus, there is continued debate among clinicians about the overall utility of these highly sensitive assays, and questions about the overall population benefits.

The diagnosis of myocardial infarction (MI) requires a characteristic dynamic profile of myocyte necrosis of cardiac biomarker(s), preferably a troponin (troponin I and T). ${ }^{2}$ There are many assays for detecting these troponins, and these vary in analytical performance. There are two defining characteristics of a highly sensitive assay - it must have a coefficient of variation (a measure of analytical imprecision) of less than $10 \%$ at the 99th percentile of a healthy reference population, and should reliably measure results accurately in at least $50 \%$ of such a population. ${ }^{5}$ A scoring system has been proposed for assays, whereby highly sensitive assays should all be deemed "guideline acceptable".,5

\section{Abstract}

Objectives: To examine differences in care and inhospital course of patients with possible acute coronary syndrome (ACS) in Australia and New Zealand based on whether a highly sensitive (hs) troponin assay was used at the hospital to which they presented.

Design, setting and patients: A snapshot study of consecutive patients presenting to hospitals in Australia and New Zealand from 14 to 27 May 2012 with possible ACS.

Main outcome measures: Rates of major adverse cardiac events (inhospital death, new or recurrent myocardial infarction, stroke, cardiac arrest or worsening heart failure); association between assay type and outcome (via propensity score matching and a generalised estimating equation [GEE]; averages of the predicted outcomes among patients who were treated with and without the availability of an hs assay (via inverse probability-weighting [IPW] with regression-adjusted estimators).

Results: 4371 patients with possible ACS were admitted to 283 hospitals. Over half of the hospitals (156 [55\%]) reported using the hs assay and most patients $(2624[60 \%])$ had hs tests $(P=0.004)$. Use of the hs assay was independent of hospital coronary revascularisation capability. Patients tested with the hs assay had more non-invasive investigations (exercise tests, stress echocardiography, stress nuclear scans, and computed tomography coronary angiography) than those tested with the sensitive assay. However, there were no differences between the groups in rates of angiography or revascularisation. All adjusted analyses showed a consistently lower rate of inhospital events, including recurrent heart failure in patients for whom the hs assay was used (GEE odds ratio, $0.75 ; 95 \% \mathrm{Cl}$, $0.60-0.94 ; P=0.014)$; IPW analysis showed a $2.3 \%$ absolute reduction in these events with the use of the hs assay $(P=0.018)$.

Conclusion: Use of hs troponin testing of patients hospitalised with possible ACS was associated with an increased rate of non-invasive cardiac investigations and fewer inhospital adverse events.

The SNAPSHOT ACS study, ${ }^{7}$ a prospective audit of the management of consecutive patients admitted with suspected ACS during a 2-week period in Australia and New Zealand, allowed a unique opportunity to assess the real-time implications of the assays used. At the time of the audit, some centres in Australia and New Zealand had access to an assay with higher sensitivity (Roche hsTnT), while many continued to use the fourth generation cTnT assay or one of various cTnI assays. In this study we aimed to explore differences in the investigation, treatment, diagnosis and inpatient clinical course for patients whose management included the use of highly sensitive versus other troponin assay results.

\section{Methods}

The SNAPSHOT ACS study was conducted from 14 to 27 May 2012 in Australia and New Zealand, and has previously been described in detail.? Briefly, it was developed as a collaborative quality improvement initiative between the Cardiac Society of Australia and New Zealand, the Heart Foundation of Australia, the Australian Commission on Safety and Quality in Health Care, the George Institute for Global Health, and health networks or state governments across Australia. Hospital participation, study protocols, recruitment, data collection and ethics approvals have been previously published.?

Over the audit period, consecutive patients having a first admission with 
a suspected or confirmed ACS were included. Patients were classified by primary discharge diagnosis into one of five diagnostic categories: (i) ST-segment-elevation MI/left bundle branch block (STEMI/LBBB); (ii) nonSTEMI (NSTEMI); (iii) unstable angina and ischaemic chest pain; (iv) unlikely ischaemic chest pain, and (v) other diagnosis.

Each site recorded the troponin assay being used (including the troponin - I or T), and the upper limit of its reference interval (clinical decision cut-point). The Roche hsTnT assay (Roche Elecsys Troponin $\mathrm{T}$ with the clinical decision cut-point of $14 \mathrm{ng} / \mathrm{L}$ ) has improved precision and meets the guideline-acceptable criteria. ${ }^{5,6,8}$ At the time of the study this was the only assay with higher precision in use in the two countries, and we refer to it in this study as the hs (highly sensitive) assay. The analytical profile of all other assays could collectively be termed sensitive or contemporary assays which were grouped into a single category termed "sensitive" assays. Hospitals that reported using both troponin $\mathrm{T}$ and troponin I assays where the upper limit of the reference interval for the troponin $\mathrm{T}$ assay was consistent with the hs assay cut-point of $14 \mathrm{ng} / \mathrm{L}$ were classified as having the hs test available.

Patients were grouped according to the binary troponin assay classification (hs assay or sensitive assay) that was used at the enrolling hospital. Patients who were subsequently transferred were analysed according to the assay used at the enrolling institution.

A common case record form was used for collating patients' characteristics, recommended therapies for ACS and inhospital events, and logistical details of patient transfers between hospitals, and hospital resources were obtained.

Inhospital events of death, new or recurrent MI, stroke, cardiac arrest or worsening heart failure were predefined (Appendix 1). Formal adjudication of events was not possible, but $2 \%-5 \%$ of all case record forms were monitored for data accuracy and quality by coordinators across all jurisdictions. Irrespective of the assay type, in accordance with the universal definition of $\mathrm{AMI}^{2}$ a change in troponin level

1 Discharge diagnoses of 4371 consecutive patients having a first admission with a suspected or confirmed acute coronary syndrome grouped by the type of cardiac troponin assays in use at the hospital to which they presented

\begin{tabular}{lcccc} 
Final diagnosis & Sensitive assay & Highly sensitive assay & Total & $P$ \\
\hline STEMI/LBBB & $156(8.9 \%)$ & $262(10.0 \%)$ & $418(9.6 \%)$ & \\
NSTEMI & $434(24.8 \%)$ & $570(21.7 \%)$ & $1004(23.0 \%)$ & \\
Unstable angina/likely ischaemic & $395(22.6 \%)$ & $530(20.2 \%)$ & $925(21.2 \%)$ & 0.004 \\
Chest pain, unlikely cardiac & $448(25.6 \%)$ & $742(28.3 \%)$ & $1190(27.2 \%)$ & \\
Other & $314(18.0 \%)$ & $520(19.8 \%)$ & $834(19.1 \%)$ & \\
Total & $1747(100 \%)$ & $2624(100 \%)$ & $4371(100 \%)$ & \\
\hline
\end{tabular}

STEMI = ST-segment elevation myocardial infarction. LBBB = left bundle branch block. NSTEMI = non-ST-segment elevation myocardial infarction.

was required to define the diagnosis of NSTEMI (Appendix 1).

\section{Statistical analysis}

We present patient characteristics, investigations, and therapies among patients surviving to hospital discharge, and inhospital events. These are tabulated as unadjusted descriptive statistics stratified by troponin assay type (hs or sensitive) in the entire patient population and among those with a final diagnosis of ACS.

As indicators of the time to care, we compared the time to angiography and the overall length of stay between hospitals performing hs assays and sensitive assays. Because these data were highly skewed, we present unadjusted comparisons only.

Two main methods were used to attempt to yield an unbiased estimate of major cardiac event risk by type of assay. One involved using propensity score matching (producing an analysis subset of 3106 successfully matched patients) and a generalised estimating equation (GEE) to evaluate the association between assay type and outcome, accommodating hospital-level clustering. Within this analysis, a propensity score of likelihood for having care provided by a hospital that had the hs assay available was developed; this included age, sex, diagnosis, Global Registry of Acute Coronary Events (GRACE) risk score, prior MI, prior coronary artery bypass grafting (CABG), atrial fibrillation, prior stroke, peripheral vascular disease, prior renal disease, prior cancer, prior dementia, need for assistance with activities of daily living, residence in a nursing home, and Australian Institute of Health and Welfare (AIHW) hospital classification. Patients from a hospital with hs assays available were matched to those in a hospital without hs assays within $\mathrm{a} \pm 2 \%$ difference in propensity score, yielding a cohort of 3106 patients (1545 and 1559 patients in the hs and sensitive troponin assay groups, respectively).

The second method involved an inverse probability-weighted (IPW) model with regression-adjusted estimators to estimate the averages of the predicted outcomes among patients who were treated with and without the availability of an hs assay for troponin in all patients. In this model, clinical and hospital variables used to estimate the probability of troponin assay type included diagnostic classification, GRACE score, glomerular filtration rate $<60 \mathrm{~mL} / \mathrm{min} / 1.73 \mathrm{~m}^{2}$, a history of diabetes and prior MI alongside metropolitan location of the hospital, an onsite cardiac service and catheter laboratory, and AIHW hospital classification. The association between troponin assay type and outcome was then further adjusted for the clinical variables described above, although no clustering on enrolling hospital was possible in this model.

A $P$ value of $<0.05$ was considered statistically significant and, as this was an exploratory analysis, no adjustment was undertaken for multiple comparisons. Analyses were undertaken using Stata version 13 (Statacorp).

\section{Results}

A total of 4398 patients were enrolled in 286 hospitals; data on troponin assay type was available for 4371 patients
1 Royal Brisbane and Women's Hospital, Brisbane, QLD.

2 Liverpool Hospital, Sydney, NSW. 3 University of Western Australia Perth, WA.

4 George Institute for Global Health, University of Sydney, Sydney, NSW

5 Concord Repatriation General Hospital Sydney, NSW.

6 Royal Melbourne Hospital, Melbourne, VIC

7 Auckland City Hospital, Auckland, New Zealand. 8 SA Health, Adelaide, SA.

9 Flinders University, Adelaide, SA

10 University of Otago, Christchurch, New Zealand. 11 Flinders Medical Centre, Adelaide, SA.

Louise.cullen@ health.qld.gov.au

doi: 10.5694/mjal3.00275 
2 Resources used for all 4371 patients* having a first admission with a suspected acute coronary syndrome (ACS) and those with (2347) and without (2024) ACS diagnoses according to the type of cardiac troponin assay used

\begin{tabular}{|c|c|c|c|c|c|c|c|c|c|}
\hline \multirow[b]{2}{*}{$\begin{array}{l}\text { Investigations and } \\
\text { treatments }\end{array}$} & \multicolumn{3}{|c|}{ ACS diagnosis } & \multicolumn{3}{|c|}{ Non-ACS diagnosis } & \multicolumn{3}{|c|}{ All patients } \\
\hline & $\begin{array}{l}\text { Sensitive } \\
\text { assay }\end{array}$ & $\begin{array}{c}\text { Highly } \\
\text { sensitive assay }\end{array}$ & $P^{\dagger}$ & $\begin{array}{l}\text { Sensitive } \\
\text { assay }\end{array}$ & $\begin{array}{c}\text { Highly } \\
\text { sensitive assay }\end{array}$ & $P^{\dagger}$ & $\begin{array}{l}\text { Sensitive } \\
\text { assay }\end{array}$ & $\begin{array}{l}\text { Highly sensitive } \\
\text { assay }\end{array}$ & $P^{\dagger}$ \\
\hline \multicolumn{10}{|l|}{ Tests } \\
\hline Exercise stress test & $54(5.5 \%)$ & $116(8.5 \%)$ & 0.005 & $97(12.7 \%)$ & $259(17.6 \%)$ & $<0.001$ & $151(8.6 \%)$ & $375(14.3 \%)$ & $<0.001$ \\
\hline Echocardiography & $318(32.3 \%)$ & $544(39.9 \%)$ & $<0.001$ & $146(19.2 \%)$ & $272(21.6 \%)$ & 0.198 & $464(26.6 \%)$ & $816(31.3 \%)$ & 0.001 \\
\hline Stress echocardiography & $14(1.4 \%)$ & $25(1.8 \%)$ & 0.439 & $12(1.6 \%)$ & $54(4.3 \%)$ & 0.001 & $26(1.5 \%)$ & $79(3.0 \%)$ & 0.001 \\
\hline Stress nuclear scan & $36(3.7 \%)$ & $29(2.1 \%)$ & 0.026 & $38(5.0 \%)$ & $45(3.6 \%)$ & 0.118 & $74(4.2 \%)$ & $74(2.8 \%)$ & 0.011 \\
\hline $\begin{array}{l}\text { Computed tomography } \\
\text { coronary angiogram }\end{array}$ & $27(2.7 \%)$ & $63(4.6 \%)$ & 0.019 & $23(3.0 \%)$ & $50(4.0 \%)$ & 0.270 & $50(2.9 \%)$ & $113(4.1 \%)$ & 0.014 \\
\hline Angiogram & $524(53.2 \%)$ & $802(58.9 \%)$ & 0.006 & $133(17.5 \%)$ & $155(12.3 \%)$ & 0.001 & $657(37.6 \%)$ & 957 (36.5\%) & 0.446 \\
\hline \multicolumn{10}{|l|}{ Procedures } \\
\hline $\begin{array}{l}\text { Percutaneous coronary } \\
\text { intervention }\end{array}$ & $279(28.3 \%)$ & $452(33.2 \%)$ & 0.012 & $5(0.6 \%)$ & $3(0.2 \%)$ & 0.146 & $284(16.3 \%)$ & $455(17.3 \%)$ & 0.349 \\
\hline Coronary artery bypass graft & $63(6.4 \%)$ & $93(6.8 \%)$ & 0.678 & $1(0.1 \%)$ & $4(0.3 \%)$ & 0.415 & $64(3.7 \%)$ & $97(3.7 \%)$ & 0.954 \\
\hline \multicolumn{10}{|l|}{ Inhospital therapy } \\
\hline Aspirin & $914(92.8 \%)$ & $1273(93.5 \%)$ & 0.523 & $598(78.5 \%)$ & $858(68.0 \%)$ & $<0.001$ & $1512(86.6 \%)$ & $2131(81.2 \%)$ & $<0.001$ \\
\hline Intravenous heparin & 319 (32.4\%) & $552(40.5 \%)$ & $<0.001$ & $52(6.8 \%)$ & $100(7.9 \%)$ & 0.363 & $371(21.2 \%)$ & $652(24.9 \%)$ & 0.006 \\
\hline $\begin{array}{l}\text { Low-molecular-weight } \\
\text { heparin }\end{array}$ & $543(55.1 \%)$ & $685(50.3 \%)$ & 0.021 & $241(31.6 \%)$ & $281(22.3 \%)$ & $<0.001$ & $784(44.9 \%)$ & $966(36.8 \%)$ & $<0.001$ \\
\hline GP IIb/IIla inhibitor & $67(6.8 \%)$ & $123(9.0 \%)$ & 0.051 & $3(0.4 \%)$ & $1(0.1 \%)$ & 0.123 & $70(4.0 \%)$ & $124(4.7 \%)$ & 0.258 \\
\hline \multicolumn{10}{|l|}{ Discharge treatment ${ }^{\ddagger}$} \\
\hline Aspirin & $823(86.6 \%)$ & $1166(87.6 \%)$ & 0.493 & $423(55.6 \%)$ & $637(50.5 \%)$ & 0.028 & $1246(72.9 \%)$ & $1800(69.8 \%)$ & 0.028 \\
\hline Other antiplatelet therapy & $585(61.6 \%)$ & $850(63.9 \%)$ & 0.266 & $128(16.8 \%)$ & $213(16.9 \%)$ & 0.963 & $713(41.7 \%)$ & 1061 (41.1\%) & 0.706 \\
\hline$\beta$-blocker & 697 (73.4\%) & 928 (69.7\%) & 0.058 & 298 (39.1\%) & 442 (35.0\%) & 0.065 & 995 (58.2\%) & 1366 (53\%) & 0.001 \\
\hline ACEi or AR2B & 674 (71.0\%) & 819 (61.5\%) & $<0.001$ & 351 (46.1\%) & $544(43.1 \%)$ & 0.194 & 1025 (60.0\%) & 1362 (52.8\%) & $<0.001$ \\
\hline Statin & 789 (84.0\%) & 1088 (81.7\%) & 0.160 & 381 (50.0\%) & 604 (47.9\%) & 0.351 & 1179 (69.0\%) & 1689 (65.5\%) & 0.017 \\
\hline $\begin{array}{l}\text { Inhospital cardiac } \\
\text { rehabilitation }\end{array}$ & 411 (43.3\%) & $545(41.0 \%)$ & 0.269 & $103(13.5 \%)$ & $110(8.7 \%)$ & 0.001 & $514(30.1 \%)$ & 654 (25.4\%) & 0.001 \\
\hline $\begin{array}{l}\text { Outpatient cardiac } \\
\text { rehabilitation }\end{array}$ & 464 (48.8\%) & 591 (44.4\%) & 0.036 & 104 (13.7\%) & $109(8.6 \%)$ & $<0.001$ & $568(33.2 \%)$ & 699 (27.1\%) & 0.036 \\
\hline
\end{tabular}

from 283 hospitals. Of these 283 hospitals, $156(55 \%)$ used an hs troponin assay (Appendix 2). Most patients presented to hospitals that used the hs assay, of which 46 (29\%) were principal referral hospitals or hospitals in major cities, with 21 (13\%) being private hospitals (Appendix 2). There was no difference in the likelihood of use of hs assays compared with sensitive assays in institutions where cardiac services, including primary percutaneous coronary intervention (PCI) were possible.

The characteristics of patients presenting to hospitals according to the assay type used are shown in Appendix 3. Patient discharge diagnoses according to the type of assay used are shown in Box 1.

Box 2 shows the use of resources for patients tested with the two groups of assays. Patients who presented to a hospital using an hs assay underwent more non-invasive investigations (eg, exercise stress tests). However, there was no difference between hospitals using the two groups of assays in the rates of angiography, PCI or CABG. Higher rates of angiography and PCI were observed among patients with a final diagnosis of ACS, and these differences persisted within the propensitymatched models (odds ratio [OR] for angiography, 1.58 ; 95\% CI, 1.16-2.14;
$P=0.003$, and OR for PCI, $1.32 ; 95 \%$ CI, $1.0-1.73 ; P=0.043$ )

Inhospital treatment for the cohort diagnosed with ACS varied in terms of the medications prescribed. Significant differences in the use of guidelinerecommended therapy ${ }^{4}$ were observed, specifically in treatment with angiotensin-converting enzyme inhibitors and $\beta$-blockers (Box 2).

Box 3 shows that 442 patients had inhospital major adverse cardiac events; this represented $9.1 \%$ of patients in hospitals using an hs assay and $11.7 \%$ of patients in hospitals using a sensitive assay $(P=0.005)$. Using the two approaches (GEE and IPW) to assessing 
3 Inhospital major adverse cardiac events, including death, myocardial infarction, cardiac arrest* in total, and new-onset heart failure stratified by the availability of highly sensitive and sensitive troponin assays (unadjusted [univariate] analysis)

\begin{tabular}{lccccccc} 
& \multicolumn{3}{c}{ Patients with an ACS diagnosis } & & \multicolumn{3}{c}{ All patients } \\
\cline { 2 - 4 } Major adverse cardiac events & Sensitive assay & Highly sensitive assay & $P$ & & Sensitive assay & Highly sensitive assay & $P$ \\
\hline Died in hospital & $35(3.6 \%)$ & $31(2.3 \%)$ & 0.065 & $38(2.2 \%)$ & $45(1.7 \%)$ & 0.275 \\
Ml/further Ml after admission & $31(3.2 \%)$ & $47(3.5 \%)$ & 0.686 & $33(1.9 \%)$ & $52(2.0 \%)$ & 0.828 \\
Inhospital heart failure & $113(11.5 \%)$ & $122(9.0 \%)$ & 0.045 & $140(8.0 \%)$ & $155(5.9 \%)$ & 0.007 \\
Total & $164(16.7 \%)$ & $187(13.7 \%)$ & 0.050 & $204(11.7 \%)$ & $238(9.1 \%)$ & 0.005 \\
\hline
\end{tabular}

ACS = acute coronary syndrome. $\mathrm{Ml}=$ myocardial infarction. $*$ Included in the total. $\bullet$

the association between assay type and outcome showed a consistently lower rate of inhospital events, including recurrent heart failure, in patients where an hs assay was used. By the GEE approach, the use of an hs troponin assay was associated with an odds ratio of 0.75 (95\% CI, $0.60-0.94 ; P=0.014)$. Similarly, by the IPW analysis, we estimated an event rate of $11.2 \%$ in patients in hospitals using sensitive troponin assays, with an average effect of a $2.3 \%$ absolute reduction in these events in patients in hospitals using the hs assay $(P=0.018)$.

Exploring the factors potentially influencing differences in outcome, we observed a shorter median delay in time to angiography in hospitals where the hs assay was available (38 hours; interquartile range [IQR], 14-72 hours for the hs assay v 43 hours; IQR, 19-75 hours for the sensitive assay; $P=0.0136$ ), but no difference in total length of stay (2.5 days; IQR, 1.1-4.8 days for the hs assay v 2.6 days; IQR, 1.1-4.9 days for the sensitive assay; $P=0.43$ ).

\section{Discussion}

The SNAPSHOT ACS registry provides several important insights into the use of highly sensitive troponin assays in the care of patients with suspected ACS.

Patients with suspected ACS who were cared for in hospitals using the hs troponin assay had a lower proportion of NSTEMI and a higher proportion of non-cardiac chest pain as final diagnoses. This contrasts with research cohorts where re-adjudication of diagnoses of individual ED patients showed an increased rate of diagnosis of NSTEMI when more highly sensitive assays were used. ${ }^{3}$ This observation may be explained by our study including more patients with suspected ACS who eventually had an alternative diagnosis (eg, arrhythmia, heart failure and pulmonary disease). The SNAPSHOT registry found a lower proportion of patients diagnosed with unstable angina in sites that used the hs assay. As elevated troponin levels are required for a diagnosis of $\mathrm{AMI}^{2}$ this finding is not surprising. However, this is the first time that the magnitude of effect of troponin assays on AMI diagnoses has been reported in an Australian and New Zealand population.

There was no pattern in the type of institution that had access to the hs assay despite the perception that large metropolitan hospitals and private facilities would have greater access to the newer assays. Patients tested with hs assays had higher rates of non-invasive investigations than those tested with the other assays. There was no difference between hospitals using the sensitive or hs assay in terms of having cardiac intensive care, echocardiography or PCI services onsite. Hospitals using the hs assay did not have higher rates of angiography or $\mathrm{PCI}$, consistent with no increase in the proportion of patients with ACS as their final diagnosis. Therefore, use of the hs assay may well drive a higher rate of some investigations, including non-invasive tests.

A significant finding was that, for patients at hospitals using the hs assay, inhospital rates of major adverse cardiac events were lower. The most substantial difference was a lower rate of inhospital heart failure in institutions where hs assays were used. This was seen in the overall population and in the ACS cohort. A modest reduction in the delay to angiography without a reduction in the length of stay was observed among patients in hospitals using hs assays. However, the availability of high-sensitivity troponin testing may lead to a larger number of patients at lower risk of ACS, or without an eventual diagnosis of ACS, being admitted. Appropriately designed randomised clinical trials informed by high-quality ACS registries are needed to determine the true incremental value of high-sensitivity troponin testing.

A number of limitations of our study must be considered. Assays may have been misclassified in terms of troponin type (I or T) and the clinical cutoffs reported. Patients were grouped according to the assay used at the hospital they presented to, and we assumed all management was informed by these results. Further, troponin testing results were not available for patients transferred from or to non-participating centres.

In conclusion, the use of highly sensitive troponin assay results in managing patients admitted with suspected ACS is associated with an increase in nonACS diagnoses with no increase in MI diagnoses. There was a lower rate of major inhospital events, predominantly heart failure, which may be attributable to the larger proportion of non-ACS diagnoses. There is an ongoing need to determine the incremental value of the widespread introduction of highly sensitive troponin assays into routine clinical practice.

Competing interests: No relevant disclosures.

Received 31 Dec 2013, accepted 27 Jun 2014 . 
1 The economic costs of heart attack and chest pain (acute coronary syndrome). Canberra: Access Economics, 2009. http://www.bakeridi. edu.au/Assets/Files/FullReport\%20-\%20 the $\% 20$ economic $\% 20$ costs $\% 20$ of $\% 20$ heart $\% 20$ attack $\% 20$ and $\% 20$ chest $\% 20$ pain\%20\%28emilable.pdf (accessed Dec 2014).

2 Thygesen K, Alpert JS, Jaffe AS, et al. Third universal definition of myocardial infarction. Circulation 2012; 126: 2020-2035.

3 Reichlin T, Twerenbold R, Reiter M, et al. Introduction of high-sensitivity troponin assays: impact on myocardial infarction incidence and prognosis. Am J Med 2012; 125: 1205-1213.

4 Chew DP, Aroney CN, Aylward PE, et al. Addendum to the National Heart Foundation of Australia/Cardiac Society of Australia and New Zealand Guidelines for the management of acute coronary syndromes (ACS) 2006. Heart Lung Circ 2011; 20: 487-502.

5 Apple FS, Collinson PO. Analytical characteristics of high-sensitivity cardiac troponin assays. Clin Chem 2012; 58: 54-61.

6 Apple FS. A new season for cardiac troponin assays: it's time to keep a scorecard. Clin Chem 2009; 55: 1303-1306.

7 Chew DP, French J, Briffa TG, et al. Acute coronary syndrome care across Australia and New Zealand: the SNAPSHOT ACS study. Med J Aust 2013; 199: 185-191.

8 Apple FS, Ler R, Murakami MM. Determination of 19 cardiac troponin I and T assay 99th percentile values from a common presumably healthy population. Clin Chem 2012; 58: 15741581. 